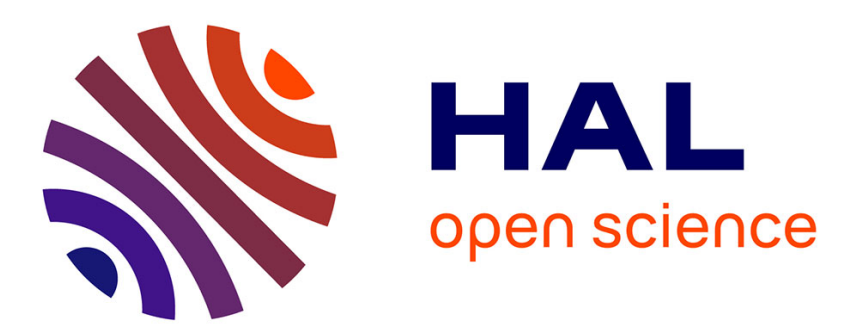

\title{
HPV-vaccination for the prevention of cervical cancer in Austria: a model based long-term prognosis of cancer epidemiology
}

\author{
Ingrid Zechmeister, Birgitte Freiesleben de Blasio, Geoff Garnett
}

\section{To cite this version:}

Ingrid Zechmeister, Birgitte Freiesleben de Blasio, Geoff Garnett. HPV-vaccination for the prevention of cervical cancer in Austria: a model based long-term prognosis of cancer epidemiology. Journal of Public Health, 2009, 18 (1), pp.3-13. 10.1007/s10389-009-0276-3 . hal-00535286

\author{
HAL Id: hal-00535286 \\ https://hal.science/hal-00535286
}

Submitted on 11 Nov 2010

HAL is a multi-disciplinary open access archive for the deposit and dissemination of scientific research documents, whether they are published or not. The documents may come from teaching and research institutions in France or abroad, or from public or private research centers.
L'archive ouverte pluridisciplinaire HAL, est destinée au dépôt et à la diffusion de documents scientifiques de niveau recherche, publiés ou non, émanant des établissements d'enseignement et de recherche français ou étrangers, des laboratoires publics ou privés. 


\title{
HPV-vaccination for the prevention of cervical cancer in Austria: a model based long-term prognosis of cancer epidemiology
}

\author{
Ingrid Zechmeister • Birgitte Freiesleben de Blasio • \\ Geoff Garnett
}

Received: 1 September 2008 / Accepted: 2 June 2009 / Published online: 11 August 2009

(C) Springer-Verlag 2009

\begin{abstract}
Aim Cervical cancer incidence and mortality have decreased for the last 20 years in Austria; however, they remain relatively high in comparison to other European countries. Screening quality has been suboptimal. In this paper we aim to predict the population-wide long-term effects on cervical cancer morbidity and mortality after introducing an HPV vaccination for 12-year-old girls (and boys) in addition to current screening in comparison with screening only.

Methods Health effects are predicted by a dynamic transmission model that was previously applied in the UK and the Norwegian contexts and validated for Austria. Outcomes analyzed are restricted to cervical cancer mortality and morbidity, which are predicted until 2060 assuming a coverage rate between $65 \%$ and $85 \%$, a duration of protection between 10 years and lifelong, and a vaccine efficacy between $80 \%$ and $100 \%$ in the base case and best case, respectively. Additionally, implications for cancer epidemiology until 2088 are estimated.

Results Compared to screening only, screening plus vaccination of 12-year-old girls (and boys) would result in a
\end{abstract}

I. Zechmeister $(\bowtie)$

Ludwig Boltzmann Institute for Health Technology Assessment,

Garnisongasse 7/20,

1090 Vienna, Austria

e-mail: Ingrid.zechmeister@hta.lbg.ac.at

B. Freiesleben de Blasio

Institute of Medical Sciences, University of Oslo,

P.O. Box 1122, Blindern 0317 Oslo, Norway

G. Garnett

Imperial College, Institute of Microparasite Epidemiology,

St. Mary's Campus, Norfolk Place,

W2 1PG London, UK median reduction of $10 \%(15 \%)$ fewer new cancer cases and $13 \%(20 \%)$ fewer cervical cancer deaths under best case assumptions over 52 years in the overall female population. In 2060 , female population-based incidence and mortality would decrease by $27 \%$ and $43 \%$, respectively, when vaccinating girls only and by $37 \%$ and $45 \%$ when additionally vaccinating boys. After 2060, a continuous further decrease in incidence and mortality can be expected with a maximum of minus $43 \%$ and $53 \%$, respectively, in 2088 when vaccinating girls only.

Conclusion Although a constant decrease in cervical cancer incidence and mortality is to be expected after introducing a population-wide HPV vaccination program in Austria, the reduction predicted by this model is lower than expected from clinical trials. This is due to several factors, such as low coverage rate and the long time horizon required for generating the maximum benefit of the vaccination in the overall population. In the context of limited resources, for further reducing cervical cancer in Austria, HPV vaccination programs need to be weighed against other public health alternatives such as improving screening quality.

Keywords HPV vaccination - Cervical carcinoma incidence $\cdot$ Cervical carcinoma mortality $\cdot$ Austria

\section{Introduction}

It has been demonstrated that infection with certain types of human papillomavirus (HPV) can cause cervical cancer. Worldwide, cervical cancer is responsible for $9 \%$ of total cancer-related mortality. However, $80 \%$ of the cases concern the so-called developing countries. In Europe there are approximately 34,000 new cases of cervical cancer each year. Around 14,000 women die each year (Ferlay et al. 
2004). In Austria there were 473 new cases of cervical carcinoma in 2003, and 169 women died from cervical cancer, representing a $2.8 \%$ share of the total cancer incidence and a $2 \%$ share of the total cancer mortality (Statistik Austria 2007b).

For the past decades the incidence and mortality of cervical carcinoma have been clearly decreasing in Western countries, including Austria. This is explained by the introduction of cytological screening (pap smear). However, in comparison with other European countries, the ageadjusted incidence and mortality rates are high in Austria (Ferlay et al. 2004). Finland is the European country with the lowest age-adjusted incidence and mortality rate: Compared to Austria, in 2002, 6.6 fewer women per 100,000 had developed the disease in Finland than in Austria, and 2.3 fewer women per 100,000 died from it (Ferlay et al. 2004). Overall, in Austria screening is opportunistic rather than organised. Potential for improvement exists in terms of screening attendance rates, particularly among women from underprivileged groups, and in terms of screening quality. Although screening is publicly financed, available data showed an annual screening participation rate of only $30 \%$ in 2005 . Participation decreases with increasing age (Hauptverband der österreichischen Sozialversicherungsträger 2007).

In addition to successful screening programs, the possibility of immunization against high-risk virus genotypes has recently been introduced. Both of the two available HPV vaccines prevent infection from 2 out of 15 identified high-risk HPV genotypes (16 and 18, detected in about $70 \%$ of cancer cases); one additionally protects against two low-risk types (6 and 11) that are related to further diseases such as genital warts. As has been shown in systematic literature reviews, the vaccines have shown high efficacy in preventing HPV-type-specific infections and precancerous lesions in clinical studies, the latter being surrogate parameters for cervical carcinoma (National Board of Health and Danish Centre for Health Technology Assessement 2007; Nielsen et al. 2007; Thiry et al. 2007). There is hope that through vaccination the number of new cervical cancers will decrease substantially. However, several important questions remain, such as duration of protection, overall reduction of cervical cancer regardless of HPV-type or possible changes in the virus dynamics.

In order to support informed decision making about whether to recommend (and finance) HPV vaccination publicly, epidemiological models that aim at long-term predictions of cervical cancer morbidity and mortality for various prevention program alternatives are a useful tool. They have been developed for several countries, often in combination with an economic evaluation (Kulasingam and Myers 2003; Sanders and Taira 2003; Goldie et al. 2004, 2007; Taira et al. 2004; Barnabas and Garnett 2005; Garnett et al. 2006; Brisson et al. 2007; Elbasha et al. 2007; French et al. 2007; Insinga et al. 2007; Kohli et al. 2007; National Board of Health and Danish Centre for Health Technology Assessement 2007; Neilson and Freiesleben de Blasio 2007; Thiry et al. 2007; Bergeron et al. 2008; Dasbach et al. 2008; Jit et al. 2008; Kim and Goldie 2008; Kulasingam et al. 2008). However, for producing reliable results, countryspecific models are required that take into account countryspecific features such as screening policy, epidemiology of the disease under evaluation and HPV prevalence.

Furthermore, many of the published studies focus on cost-effectiveness results without presenting populationbased epidemiological predictions, or they analyze health benefits for a single vaccinated cohort only. However, from a public health perspective as well as from a decisionmaker's view, it is equally relevant how cancer epidemiology may change population-wide for a defined time horizon after introducing a vaccination program. In the following article we aim at addressing this question. We present the results of an epidemiological long-term prognosis for cervical carcinoma in Austria for a specified time period assuming different prevention strategies.

\section{Method}

\section{Prevention strategies}

We analyzed three different prevention strategies: (1) 'screening only' (the current standard treatment); (2) vaccination of 12-year-old girls in addition to screening (HPV F); (3) vaccination of 12-year-old girls and boys in addition to screening (HPV F+M). The outcome parameters of interest were cervical carcinoma incidence and mortality. The time horizon in the base case analysis was 52 years (from 2008 to 2060). In an additional analysis we extrapolated the results to the year 2088 .

\section{Dynamic model structure}

To predict long-term effects, a dynamic compartmental model developed and applied in the UK and in Norway (Neilson and Freiesleben de Blasio 2007) was adapted and validated for Austria. Although the complexity of a dynamic model limits transparency, this type of model was chosen to account for herd immunity and to demonstrate the health effect for women from vaccinating boys.

The model follows a population through HPV infection, chronic cervical infection and cervix cancer development based on the natural history of cervical carcinogenesis. The model simulates HPV transmission, the occurrence of precancerous lesions $(\mathrm{CIN})$ and the development of cervical carcinoma (see Table 1 in the next section for progression 
Table 1 Model parameters for the dynamic model

\begin{tabular}{|c|c|c|}
\hline Parameter & Value $^{\mathrm{a}}$ & Source \\
\hline \multicolumn{3}{|l|}{ Natural history of disease } \\
\hline $\begin{array}{l}\text { Progression to chronic infection } \\
\text { (type } 16, \text { type } 18, \text { type other) }\end{array}$ & $0.1 ; 0.085 ; 0.085$ & (Goldie et al. 2004; Kim et al. 2007) and references there \\
\hline $\begin{array}{l}\text { Recovery from transient infection } \\
\text { (type } 16, \text { type } 18 \text {, type other) }\end{array}$ & $0.9 ; 1.3 ; 1.9$ & Initial estimates based on (Ho et al. 1998; Muñoz et al. 2004b) \\
\hline $\begin{array}{l}\text { Progression by stage (cin } 1, \text { cin } 2, \\
\text { cin } 3 \text {, cis, ccI, ccII,ccIII, ccIV) }\end{array}$ & $\begin{array}{l}1.7 ; 0.03 ; 0.045 ; 0.15 ; 0.18 \\
0.20 ; 0.20\end{array}$ & (Goldie et al. 2004; Kim et al. 2007) and references there \\
\hline Regression by stage $(\operatorname{cin} 1, \operatorname{cin} 2, \operatorname{cin} 3)$ & $0.37 ; 0.1 ; 0.04$ & (Goldie et al. 2004; Kim et al. 2007) and references there \\
\hline $\begin{array}{l}\text { Relative regression } \\
\text { cin1 to normal }\end{array}$ & 1.0 & (Kim et al. 2007) \\
\hline $\operatorname{cin} 2$ to $(\operatorname{cin} 1$, normal $)$ & $0.7 ; 0.3$ & \\
\hline $\operatorname{cin} 3($ to $\operatorname{cin} 2, \operatorname{cin} 1$, normal) & $0.7 ; 0.15 ; 0.15$ & \\
\hline Age-specific progression rate & $0.175 ; 0.6 ; 0.9 ; 0.9 ; 0.9 ; 1.2$ & Fitted to Austrian data (Hackl 2007) \\
\hline $\begin{array}{l}\text { Recovery from cancer by stage } \\
\text { (cis, ccI, ccII, ccIII, cIV) }\end{array}$ & $0.35 ; 0.25 ; 0.2 ; 0.1 ; 0.1$ & $\begin{array}{l}\text { Initial estimates based on Norwegian data; subsequently } \\
\text { fitted to Austrian data (Hack1 2007) }\end{array}$ \\
\hline $\begin{array}{l}\text { Death rate from CC by stage } \\
\text { (cis, ccI, ccII, ccIII, cIV) }\end{array}$ & $0.05 ; 0.2 ; 0.5 ; 0.85 ; 1.0$ & $\begin{array}{l}\text { Initial estimates based on Norwegian data; subsequently } \\
\text { fitted to Austrian data (Hack1 2007) }\end{array}$ \\
\hline $\begin{array}{l}\text { Fraction of slow progressors } \\
\text { (type } 16 \text {; type } 18 \text {; type other) }\end{array}$ & $0.96 ; 0.945 ; 0.985$ & Assumption \\
\hline $\begin{array}{l}\text { Relative type-specific progression } \\
\text { (type } 16 \text {; type } 18 \text {; type other) }\end{array}$ & $1.0 ; 1.05 ; 0.8$ & Fitted to model \\
\hline $\begin{array}{l}\text { Hysterectomies by age } \\
\text { Age group } 1-6\end{array}$ & $\begin{array}{l}5 e-4 ; 5 e-4 ; 5 e-4 ; 5 e-4 \\
5 e-4 ; 5 e-4\end{array}$ & UK data from Geoff Garnett \\
\hline Loss of natural immunity & 0.05 & Assumption \\
\hline $\begin{array}{l}\text { Transmission rate } \\
\text { (Type 16; type } 18 \text {; type other) }\end{array}$ & $0.6 ; 0.1 ; 0.3$ & Fitted to model; initial estimates from Geoff Garnett \\
\hline $\begin{array}{l}\text { Type-specific incidence of cervical cancer } \\
\text { (type 16; type } 18 \text {; type other) }\end{array}$ & $0.55427 ; 0.15376 ; 0.29197$ & (Muñoz et al. 2004a) \\
\hline Relative type-specific precancerous lesions & $58-59 \% ; 41-42 \%$ & (Roberts et al. 2006) \\
\hline
\end{tabular}

$$
\begin{aligned}
& \text { (CIN 2/3) } \\
& \text { (Type } 16+18: \text { type other) }
\end{aligned}
$$

\section{Screening}

Fraction of women screened

Period of implementation

Base case 0.3

1989-1992

Relative screening rate by age group

Sensitivity by stage of pathogenesis ( $\operatorname{cin} 1$; cin2; cin3; cis; ccI-IV)

Relative screening rate by sexual

activity and vaccination

(low, medium, high, vaccination)

Vaccination

$0.96 ; 1.44 ; 1.32 ; 1.13$;

$0.95 ; 0.64$

$0.50 ; 0.63 ; 0.64 ; 1.0 ; 1.0$

$0.5 ; 0.5 ; 0.5 ; 0.5$

Austrian data (Hauptverband der österreichischen Sozialversicherungsträger 2007)

Austrian data (Vutuc et al. 1999)

Austrian data (Hauptverband der österreichischen Sozialversicherungsträger 2007)

(Coste et al. 2003)

Assumption

Efficacy

Base case 0.90

Base case 0.65

$(0.5-0.85)$

Screening rate of vaccinated women

Waning efficacy/duration of protection

Susceptibility of vaccines (type 16;

type 18 ; type other)

Sexual behavior

Fraction of activity groups

(low; medium; high)

$1.0 ; 1.0 ; 0.0$
0.3

Base case

$0.0118 / 10$ years

$0.869 ; 0.125 ; 0.006$
Austrian data (Bundesministerium für Gesundheit Familie und Jugend (BMGFJ) 2007)

Austrian data (Hauptverband der österreichischen

Sozialversicherungsträger 2007)

Assumption

(Nielsen et al. 2007)

Guided by National Survey of Sexual Attitudes and Lifestyles II (UK, 2000) and survey of sexual behavior in Norway, 2002 
Table 1 (continued)

\begin{tabular}{|c|c|c|}
\hline Parameter & Value $^{a}$ & Source \\
\hline Relative activity (high; medium; low) & $37.0 ; 8.5 ; 1.0$ & $\begin{array}{l}\text { Guided by National Survey of Sexual Attitudes and } \\
\text { Lifestyles II (UK, 2000) and survey of sexual behavior } \\
\text { in Norway, } 2002\end{array}$ \\
\hline $\begin{array}{l}\text { Activity by age } \\
\text { (Age group 1-6) }\end{array}$ & $\begin{array}{l}6.0 ; 3.0 ; 2.0 ; 1.5 \\
1.0 ; 0.8\end{array}$ & $\begin{array}{l}\text { Guided by National Survey of Sexual Attitudes and } \\
\text { Lifestyles II (UK, 2000) and survey of sexual behavior } \\
\text { in Norway, } 2002\end{array}$ \\
\hline $\begin{array}{l}\text { Acts per partnership } \\
\text { High-high; high-med; high-low }\end{array}$ & $2 ; 12 ; 104$ & \multirow{3}{*}{$\begin{array}{l}\text { Guided by National Survey of Sexual Attitudes and } \\
\text { Lifestyles II (UK, 2000) and survey of sexual behavion } \\
\text { in Norway, } 2002\end{array}$} \\
\hline Med-med, med-low & $12 ; 104$ & \\
\hline Low-low & 104 & \\
\hline Average partner change rate & 0.8 & $\begin{array}{l}\text { Guided by National Survey of Sexual Attitudes and } \\
\text { Lifestyles II (UK, 2000) and survey of sexual behavior } \\
\text { in Norway, 2002 }\end{array}$ \\
\hline
\end{tabular}

a: Rates unless stated otherwise; ccI: cervical carcinoma stage I; cin: cervical intraepithelial neoplasia; cis: carcinoma in situ

rates). Figure 1 presents the model structure and the way it reflects the natural history of disease.

The model consists of a set of coupled differential equations that are updated on a weekly basis; the new compartment sizes are estimated using a fourth-order Runge-Kutta method. The first 100 years are run without intervention to let the model reach dynamic equilibrium.

The initial population is assumed equal to the current Austrian population with a flat age structure. The birth rate is constant, and no immigration or emigration is considered during the simulation period. The model divides individuals into mutually exclusive compartments based on their values of state parameters. Annual cohorts up to the age of sexual

\section{Model structure}

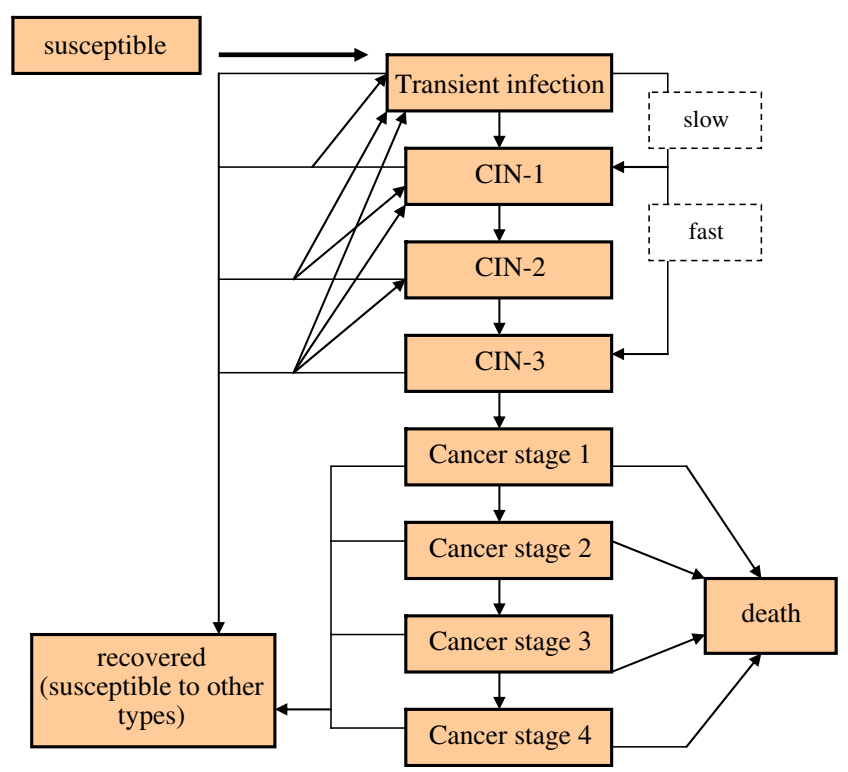

Fig. 1 Model structure for the natural history of disease. CIN: Cervical intraepithelial neoplasia debut (assumed at the age of 15) are simulated allowing for age-specific vaccination. In the current analysis vaccination is at the age of 12. Furthermore, the model handles six '10year age groups' at risk of infection and cancer up to the age of 74 . This means that outcome predictions are restricted to persons aged 15 to 74 . As a considerable proportion of cervical carcinoma incidence und mortality occurs after the age of 74 , the model underestimates the possible benefit of the vaccination after 2060. While the model in general handles four groups of viral types (HPV 16, HPV 18, HPV 6/11 and 'other oncogenic types'), the current analysis focuses on targeting the high-risk types 16 and 18 only. In the model there are three vaccine statuses: 'unvaccinated,' 'protected' and 'vaccinated with loss of protection.' Virus transmission is modeled through sexual mixing, which is divided into three activity classes with low, medium or high sexual activity defined by the changes in partnerships per year (see Table 1 for details). Mixing between individuals depends on their age and activity class. The resulting number of sexual acts per partnership is included into the transmission dynamic.

Persons who recover from infection develop typespecific natural immunity, which is lost at a constant rate within a given time period. During this time span persons are, however, susceptible to other HPV types.

Infection can result in precancerous lesions that can finally lead to invasive cancer. On the other hand, regression from more severe to less severe stages of precancerous lesions is possible. Table 1 shows the rates for progression and regression applied.

Input parameters

Table 1 demonstrates the values for all the input parameters used in the model as well as the data sources. The values for the input parameters are either based on published 
literature or on Austria-specific data. For some currently unknown parameters such as duration of protection, we had to make assumptions that were varied in sensitivity analysis.

We used Austria-specific screening data that show an annual screening coverage of around $30 \%$. Yet, as mentioned above, participation varies highly depending on the age group, with older women showing a particularly low attendance. This was acknowledged in the age-specific screening parameters. It was assumed that screening remains unchanged in both vaccinated and unvaccinated women.

Furthermore, vaccine efficacy in the base case was $90 \%$ against HPV-type-specific infection (based on clinical trial data). Vaccination coverage for a three-dose vaccination in 12 year olds was assumed to be $65 \%$. This is based on the average experience from other publicly financed vaccination programs in Austria where a coverage of only 50\% in school-based publicly funded vaccination programs and of $85 \%$ in public vaccination programs for newborns was shown [Bundesministerium für Gesundheit Familie und Jugend (BMGFJ) 2007]. Finally, because duration of vaccine protection is unknown, we assumed waning vaccine efficacy, and a booster vaccination was implemented after 10 years in the base case (see Table 1 for details): During a 10-year interval the model assumes that $\sim 11 \%$ of the women who were vaccinated and have gained immunity have lost it again. This value grows with time, so that 50 years after the initial vaccination $\sim 55 \%$ of the women who acquired immunity through vaccination are still protected. When a booster vaccination is applied, the waning process is assumed similar to the one described above. However, as the model program works with age groups and therefore does not distinguish between individual ages within groups, for example, a person aged 15 and one aged 24, we had to 'distribute' the booster among the entire age group. Assuming that HPV prevalence declines with age, the effects from the booster will be on the optimistic side. Overall, parameters and assumptions in the base case are conservative. They were, however, varied in the sensitivity analysis.

Model results were used for calculating population-wide changes in epidemiology by applying data on demography predictions from Austria (Statistik Austria 2007a).

\section{Validation}

For model calibration, the output of the model was fitted to the age-specific cervical carcinoma incidence and mortality data from Austria (Hackl 2007). For calibrating the incidence of type-specific high-grade lesions and cervical carcinomas, international data were used (Muñoz et al. 2004a; Roberts et al. 2006) (see Table 1). In terms of validity, we evaluated the predictive validity of the model by comparing the model results with observed age-specific and overall Austrian cervical cancer incidence and mortality data for the years 2001 to 2006 .

\section{Sensitivity analysis}

To test for uncertainty all key assumptions from the base case were varied in various one-way sensitivity analyses. We modified vaccination coverage between $50 \%$ and $85 \%$, and we assumed different durations of protection up to lifelong protection. Moreover, vaccine efficacy in terms of protection from persistent infections was varied between $80 \%$ and $100 \%$. Finally, we conducted a multivariate sensitivity analysis where we defined a best case scenario (100\% efficacy, no waning, $85 \%$ vaccination coverage) and ran the model under these assumptions for either vaccinating girls only or vaccinating girls and boys.

\section{Results}

Model validity

Model outputs for cervical carcinoma incidence and mortality are in accordance with Austrian epidemiological data. Incidence and mortality rates between 2001 and 2006 in the model do not differ more than $15 \%$ from the actual rates in Austria. If at all, the model slightly overestimates the rates. However, as Austrian cervical carcinoma epidemiological data seem to slightly underestimate the true burden of the disease, this in fact supports external validity of the model outputs. Figure 2 demonstrates the validation results for observed total cervical carcinoma incidence and mortality for those years where data were available compared with the model results. Similar to overall incidence and mortality, age-specific incidence and mortality predicted by the model are consistent with observed data.

Epidemiological impact of different HPV vaccination strategies

Tables 2 and 3 show the results for cervical carcinoma incidence and mortality predictions in Austria until 2060 for women aged 15 to 74 for the different prevention strategies and for the base case as well as for the best case scenarios.

Compared to screening only, vaccination of 12-year-old girls would result in a constant reduction of cervical carcinoma incidence and mortality over the next 52 years. Due to the natural history of cervical carcinoma development out of precancerous lesions, reduction starts to become visible after 20 years. In the year 2060, compared 
Fig. 2 Validation results for cervical carcinoma incidence and mortality: comparison between observed and predicted epidemiology (2001 to 2006); source: (Hackl 2007)

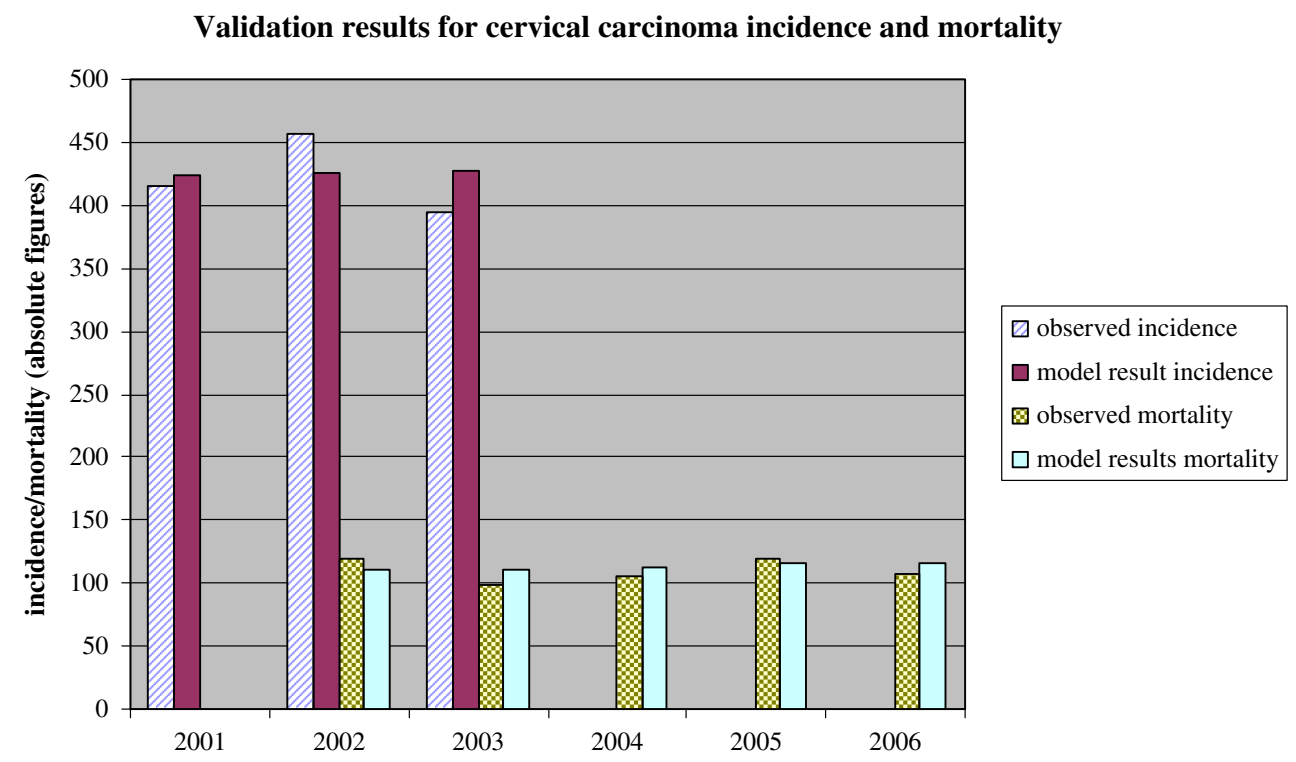

to screening only, minus $23 \%$ incidence cases and minus $27 \%$ cervical carcinoma deaths would have to be expected under the base case assumptions (coverage rate of $65 \%$, 90\% efficacy against persistent HPV 16/18 infection and waning efficacy including a booster in year 10). On average, incidence and mortality would be reduced by $9 \%$ and $11 \%$, respectively, over 52 years. This equals roughly 2,240 fewer new cancer cases and 770 fewer deaths compared to screening only. However, 22,000 women in Austria would still develop cervical cancer and about 6,000 would die over 52 years.

Vaccinating girls and boys increases the reduction of incidence and mortality to minus $33 \%$ and minus $39 \%$ in 2060 , which corresponds to $14 \%$ fewer new cases and $18 \%$ fewer deaths on average over 52 years. In absolute numbers 3,440 new cases and 1,200 deaths could be prevented by the vaccination program compared to screening only, while 20,800 women would still develop cervical cancer, and 5,500 would die despite introducing the vaccination program.

Figure 3 demonstrates the results of the one-way and multivariate sensitivity analyses for the case of vaccinating girls only. In a best case scenario $(85 \%$ coverage, $100 \%$ vaccine efficacy, life-long protection) reductions of incidence and mortality would increase further. When vaccinating girls only, in $206027 \%$ fewer new cancer cases and $32 \%$ fewer cancer deaths compared to screening only can be expected. Vaccination of girls and boys under best case assumptions increases reductions further. While over 52 years in the 'HPV F-scenario' 2,500 (10\%) new cases and $870(13 \%)$ deaths would be prevented, compared to screening only, in the 'HPV F + M-scheme' the number of prevented new cases and deaths rises to almost 3,700 (15\%) and $1,330(20 \%)$, respectively (figures not shown). This corresponds to an overall reduction in incidence rate until
2060 of 1.4 per 100,000 women when vaccinating girls and minus 2 per 100,000 women when vaccinating girls and boys. Accordingly, reduction in mortality rates would be expected at minus 0.5 per 100,000 women when vaccinating girls only and minus 0.7 per 100,000 women when additionally vaccinating boys.

Furthermore, the model demonstrates that increasing the coverage rate to $85 \%$ while leaving all other assumptions from the base case (booster in year ten, $90 \%$ vaccine efficacy) unchanged is almost as effective as the best case scenario where, in addition to a $85 \%$ coverage rate, lifelong protection and a slightly higher vaccine efficacy were assumed.

As may be anticipated, more pessimistic scenarios, such as reducing the coverage rate to below $60 \%$ or assuming waning vaccine efficacy without a booster, result in lower health benefits (see Fig. 3). For example, in the case of waning efficacy without giving a booster, the model predicts a reduction of $17 \%$ and $19 \%$ in cervical cancer incidence and mortality, respectively, in 2060 when vaccinating girls only. Over 52 years 700 more women would develop cancer and 240 more women would die of cervical cancer than under base case assumptions where a booster is assumed.

Since generating the maximum benefit of HPV vaccination will require a considerably longer time span than 52 years, the results have been further extrapolated to the year 2088. Figure 4 shows the expected reduction in incidence and mortality compared to screening only for Austria given all other circumstances remain unchanged. As the figure demonstrates, further health gains can be expected in the long run. However, even in 2088 (80 years after introduction of the vaccination program) reduction in population-wide incidence does not exceed minus $43 \%$, and mortality reduction could at best be expected at minus $53 \%$. 


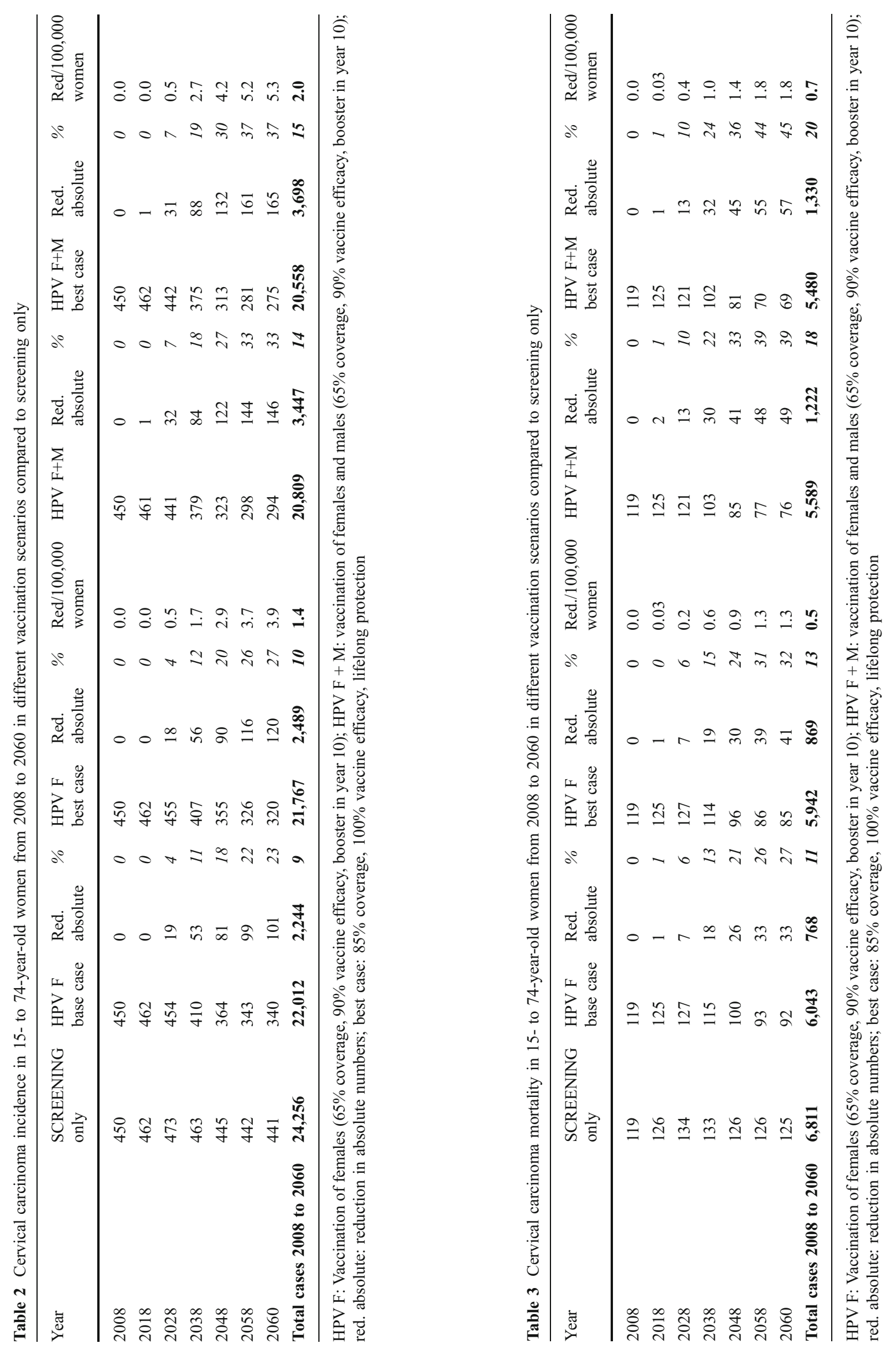


Fig. 3 Reduction in carcinoma incidence and mortality in 2060 in sensitivity analyses (girls only scenarios)

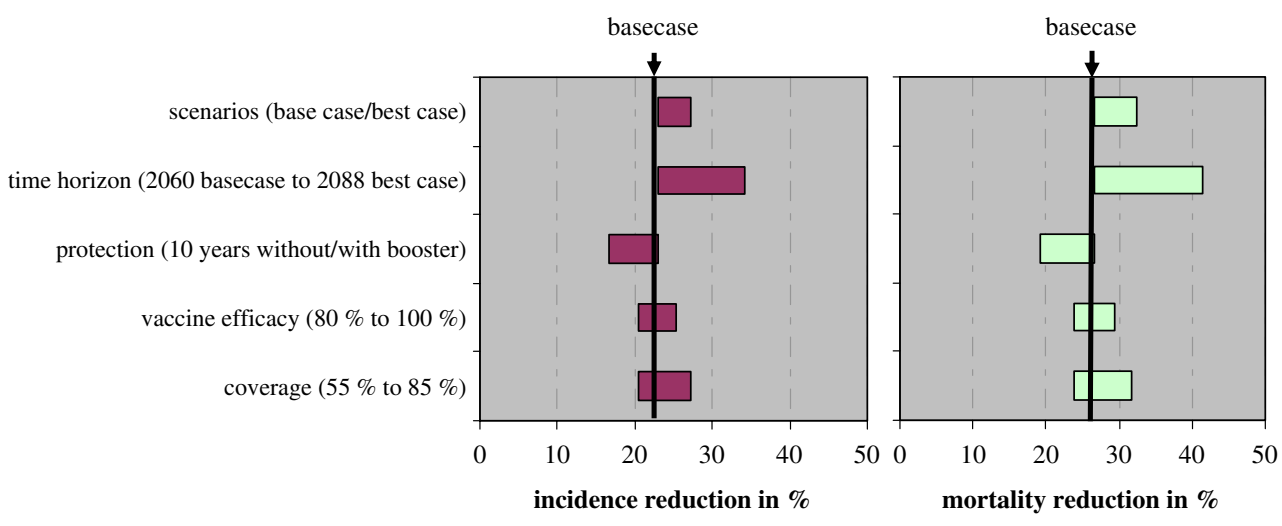

\section{Discussion}

Results from clinical HPV vaccination trials have shown a very favorable vaccine efficacy with respect to preventing HPV 16/18 infections and HPV 16/18 precancerous lesions. Since these two genotypes have been observed in at least $70 \%$ of cervical carcinomas, a reduction of cervical cancer close to $70 \%$ has been announced by the vaccine developers. In contrast to models that have analyzed long-term effects in vaccinated cohorts only, the strength of our approach is that it provides information on the overall public health impact with respect to cervical cancer within a defined time period, which is likely to be of relevance for decision makers. The model results for the Austrian case show that viewed from an overall epidemiological perspective, reduction in cervical cancer incidence and mortalityat least within the next 50 years - lies considerably below the expected magnitude.
This can be explained by several reasons. Firstly, even in the best case, vaccination coverage rate and vaccine efficacy will be below $100 \%$ in a real-life situation. This hinders virus elimination, which is generally difficult to achieve in sexually transmitted viruses (Garnett 2005). Secondly, it takes a long time to benefit from the vaccination with respect to cervical cancer because of the long time span the cancer takes to develop. Hence, in 50 years time many women who would have been vaccinated by then would not have had 'the chance' to accrue the benefits. The main reason, however, is that introducing a vaccination program, even if populationwide, means that there will still be many unvaccinated (older) women whose risk of developing cancer remains unchanged provided that the current screening praxis is being continued. In other words, a large proportion of the demonstrated cancer (mortality) cases will occur due to the HPV infections in women who are older than 12 in 2008
Reduction in cervical carcinoma incidence in \% 2018 to 2088

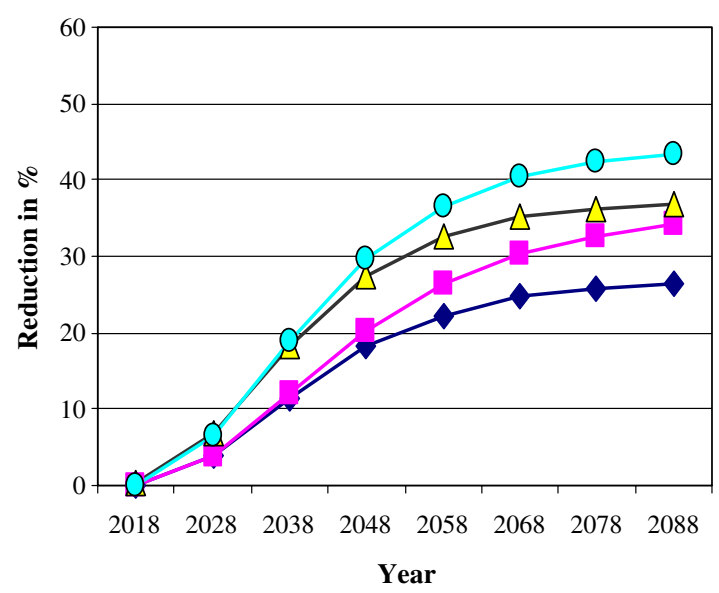

Fig. 4 Reduction in cervical carcinoma incidence and mortality in \% from 2018 to 2088. HPV F: Vaccination of females (65\% coverage, $90 \%$ vaccine efficacy, booster in year 10$)$; HPV F + M: vaccination of
Reduction in cervical carcinoma mortality in \% 2018 to 2088

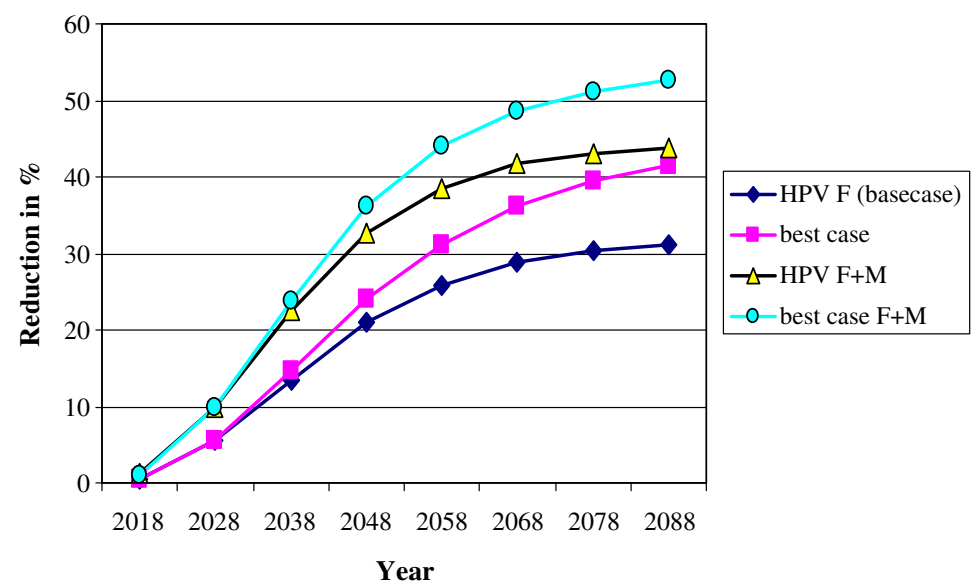

females and males (65\% coverage, $90 \%$ vaccine efficacy, booster in year 10 ); best case: $85 \%$ coverage, $100 \%$ vaccine efficacy, lifelong protection 
and who would not be vaccinated. Part of these cases may be reduced by introducing a catch-up program, which has shown favorable results in other studies (e.g., the National Board of Health and Danish Centre for Health Technology Assessement 2007). As this alternative was not considered by the Austrian decision makers, it was not addressed in the model but may be interesting to focus on in further research.

As has been shown in the sensitivity analysis, under the assumption of an $85 \%$ coverage rate ( + booster in year 10 and $90 \%$ vaccine efficacy), reductions of incidence and mortality are almost as high as under best case assumptions where, in addition to the former, lifelong duration of protection and a slightly higher vaccine efficacy have been assumed. This can be explained by several factors. Firstly, the coverage rate seems to be more influential than other factors such as vaccine efficacy. Secondly, as has been mentioned earlier, the booster in the ' $85 \%$ coverage scenario' tends to slightly overestimate the health benefits because of the model characteristics. In other words, the booster more than compensates for the assumed loss of efficacy, and the effects are gained early in time. As a consequence, under the assumption of lifelong protection, health gains with respect to reduced mortality are slightly lower than in the ' $85 \%$ coverage scenario.' In the long run, however, the difference diminishes.

Our results differ from other studies that have shown more favorable effects (Goldie et al. 2004; Barnabas and Garnett 2005; Elbasha et al. 2007; French et al. 2007; Insinga et al. 2007; Kohli et al. 2007; Dasbach et al. 2008). This is firstly due to the different research question in our study, which addresses population-wide benefits for a given time period rather than health effects for vaccinated cohorts. Furthermore, some studies addressed the epidemiological impact for HPV 16/18-related carcinomas only (Barnabas and Garnett 2005; French et al. 2007) or evaluated epidemiological changes for a 100 -year time frame, while we addressed the impact on total cancer incidence and mortality for a maximum of 80 years. Another reason for differences in results is that the situation in Austria generally differs from other countries, especially in terms of screening quality and baseline epidemiology. For instance, in the scenario 'vaccination of 12-year-old girls,' the low screening participation rate (in unvaccinated women) will result in higher total incidence and mortality rates in Austria than in countries with higher screening participation. This may for example be one reason that the reduction in cervical cancer in the Elbasha et al. (2007) base case is higher than in ours. Furthermore, in most of the studies assumptions concerning vaccination have been more optimistic than in ours, particularly with respect to vaccination coverage, vaccine efficacy and duration of protection (e.g. Elbasha et al. 2007), while we chose a conservative approach. Finally, in contrast to other studies
(Goldie et al. 2004; Elbasha et al. 2007), our analysis has been restricted to invasive cervical carcinoma only, thus not demonstrating the impact of vaccination on precancerous lesions or on other (rare) HPV-related diseases (such as vulval cancer).

On the other hand, other studies have also presented less favorable results when considering less optimistic assumptions in sensitivity analyses. For example, Elbasha et al. (2007) demonstrated a long-term reduction of new cervical carcinomas of $28 \%$ per year when choosing a vaccine protection of 10 years only compared to $91 \%$ reduction in their most effective strategy. Additionally, studies that particularly take into account the difficulties of preventing a sexually transmitted infection (e.g., Hughes et al. 2002) have also shown less encouraging results.

As has been mentioned in the introduction, further studies on HPV vaccination exist that focus on economic evaluation. We do not discuss their results here because we restricted our paper to epidemiological outputs only. However, this issue has been addressed in a separate paper (Zechmeister et al. 2009).

Although the focus on invasive carcinoma only is justified by its highest burden of disease within the HPVrelated diseases and by the fact that the prevention of cervical carcinoma has been one of the main arguments brought forward by the proponents of the vaccination, this is at the same time the main limitations of the study. Most importantly, since precancerous lesions contribute substantially to the costs and burden of disease, evaluating them as a separate outcome parameter would make additional health benefits of the vaccine visible and benefits observable earlier. On the other hand, some factors that may have a contrary effect have also not been taken into account in the model. These are, for example, the immigration of unvaccinated persons, possible changes in virus dynamics or in the risk behavior of vaccinated people. For instance, we do not know whether the behavior of vaccinated women in terms of screening and safer sex will change and in which way this change will occur.

Further limitations of the study are related to data restrictions. Although Austrian data have been used to populate the model wherever possible, such data were not available for some parameters such as the sexual activity. Thus, they were based on UK and Norwegian survey data that were used in the original model. Although these countries have a cultural context similar to Austria, we do not know how exactly they reflect the sexual behavior of Austrians.

Finally, the predictions for the period after 2060 face increased uncertainty since, firstly, demographic data were not available until 2088, and predictions for such a long time horizon generally have to be treated with caution. Secondly, as mentioned in the methods section, the model 
only predicts results for women aged 15 to 74 . In the prediction up to 2088, an increasing proportion of women will be older than 74 years. Potential cases avoided in these women are not shown by the model, and therefore the model underestimates the benefits in the extended scenario.

The study has raised some important issues for further research. For example, it is likely that due to the reduction in the incidence of precancerous lesions, changes in screening policy will be required. As has been done by others (e.g., Kulasingam and Myers 2003; Goldie et al. 2004), modeling could as well be used to analyze the impact of different screening strategies. Not least, more detailed sensitivity analysis will be required in future research for further uncertain parameters such as natural immunity or sexual activity.

\section{Conclusion}

The study has demonstrated that a population-wide vaccination program against HPV 16/18 would in the long run result in a further decrease of cervical carcinomas in Austria. However, due to the nature of the disease and the apparent difficulty of high vaccination coverage in Austria, after 50 years of vaccination, the health gains in terms of overall population health will be far from the expected maximum benefit.

In the context of increasing budget constraints, introducing a publicly financed HPV vaccination program needs to be weighed against other possible (cheaper) alternatives, the more so as the epidemiologic relevance of cervical cancer is low in Austria compared to other types of cancer in women (e.g., breast cancer, lung cancer). Since there is considerable room for improvement in screening quality, a reduction of cervical cancer may also be achieved by a better organized screening system at lower costs, leaving more resources for other preventive measure for more pressing health problems. This needs to be addressed in further research, particularly in economic evaluations. However, it needs to be born in mind that screening is a suboptimal secondary prevention measure in contrast to primary prevention with vaccination.

As a number of new vaccines for increasingly less virulent or prevalent diseases are currently in development and health-care budgets come ever more under pressure, more thoughts need to be spent on how to support rational vaccination policy that systematically takes into account epidemiological, health economic, organizational and equity criteria (Piso and Wild 2008).

Acknowledgement The article is based on the results of the report entitled 'Economic evaluation of HPV vaccination in Austria,' which was funded by the Austrian Ministry of Health. We are grateful to the people from various Austrian institutions (Statistics Austria, Ministry of Health, Federation of the Austrian Social Insurance Institutions, Tyrolean Hospital Association) who provided the numerous data required, to the medical experts who supported us with their advice and expertise, and to our colleagues Claudia Wild and Philipp Radlberger who helped us with data collection and finalizing the analysis.

Conflict of Interest The authors confirm that there are no relevant associations that might pose a conflict of interest.

\section{References}

Barnabas RV, Garnett GP (2005) The potential public health impact of vaccines against human papillomavirus. In: Prendiville W, Davies P (eds) The health professionals HPV handbook. Taylor \& Francis, Abingdon

Bergeron C, Largeron N, McAllister R, Mathevet P, Remy V (2008) Cost-effectiveness analysis of the introduction of a quadrivalent human papillomavirus vaccine in France. International Journal of Health Technology Assessment in Health Care 24:10-19

Brisson M, Van der Velde N, De Wals P, Boily M-C (2007) The potential cost-effectiveness of prophylactic human papillomavirus vaccines in Canada. Vaccine 25:5399-5408

Bundesministerium für Gesundheit Familie und Jugend (BMGFJ) (2007) Impfstatistik. In. Bundesministerium für Gesundheit, Familie und Jugend; Abteilung Infektionskrankheiten, Seuchenbekämpfung und Krisenmanagement, Wien

Coste J, Cochand-Priollet B, de Cremoux P, Le Galés C, Cartier I, Molinié V, Labbé S, Vacher-Lavenu M-C, Vielh P (2003) Cross sectional study of conventional cervical smear, monolayer cytology, and human papillomavirus DNA testing for cervical cancer screening. Br Med J 326:733

Dasbach EJ, Insinga R, Elbahsa EH (2008) The epidemiological and economic impact of a quadrivalent human papillomavirus vaccine $(6 / 11 / 16 / 18)$ in the UK. BJOG. An International Journal of Obstetrics and Gynaecology 115:947-956

Elbasha EH, Dasbach EJ, Insinga RP (2007) Model for assessing human papillomavirus vaccination strategies. Emerg Infect Dis 13:28-41

Ferlay J, Bray F, Pisani P, Parkin DM (2004) GLOBCAN 2002 cancer incidence. mortality and prevalence worldwide. In: IARC CancerBase No. 5 version 2.0. IARC Press, Lyon

French KM, Barnabas RV, Lehtinen M, Kontula O, Pukkala E, Dillner J, Garnett GP (2007) Strategies for the introduction of human papillomavirus vaccination: modelling the optimum age- and sex-specific pattern of vaccination in Finland. $\mathrm{Br} \mathrm{J}$ Cancer 96:514-518

Garnett GP (2005) Role of herd immunity in determining the effect of vaccines against sexually transmitted disease. J Infect Dis 191: s97-s106

Garnett GP, Kim JJ, French K, Goldie SJ (2006) Modelling the impact of HPV vaccines on cervical cancer and screening programmes. Vaccine 24S3:178-186

Goldie SJ, Kohli M, Grima D, Weinstein MC, Wright TC, Bosch FX, Franco E (2004) Projected clinical benefits and cost-effectiveness of a human papillomavirus $16 / 18$ vaccine. J Natl Cancer Inst 96:604-615

Goldie SJ, Kim JJ, Kobus K, Goldhaber-Fiebert JD, Salomon J, O'Shea MKH, Bosch X, de Sanjosé S, Franco E (2007) Cost-effectiveness of HPV 16, 18 vaccination in Brazil. Vaccine 25:6257-6270

Hackl M (2007) Krebsstatistik. Sonderauswertung. In: Statistik Austria, Wien

Hauptverband der österreichischen Sozialversicherungsträger (2007) Datenerhebung Pap-Abstrich. In: Hauptverband der österreichischen Sozialversicherungsträger, Wien 
Ho GY, Bierman R, Beardsley L, Chang CJ, Burk RD (1998) Natural history of cervicovaginal papillomavirus infection among cytologically normal women. N Eng J Med 338:423-428

Hughes JP, Garnett GP, Koutsky LA (2002) The theoretical population-level impact of a phrophylactic human papilloma virus vaccine. Epidemiology 13:631-639

Insinga R, Dasbach EJ, Elbahsa EH, Puig A, Reynales-Shigematsu LM (2007) Cost-effectiveness of quadrivalent human papillomavirus (HPV) vaccination in Mexico: a transmission dynamic model-based evaluation. Vaccine 26:128-139

Jit M, Choi YH, Edmunds WJ (2008) Economic evaluation of human papillomavirus vaccination in the United Kingdom. Br Med J 337:a769

Kim J, Goldie S (2008) Health and economic implications of HPV vaccination in the United States. N Eng J Med 359:821-832

Kim JJ, Kuntz KM, Stout NK, Mahmud S, Villa LL, Franco E, Goldie S (2007) Multiparameter calibration of a natural history model of cervical cancer. Am J Epidemiol 166:137-150

Kohli M, Ferko N, Martin A, Franco E, Jenkins D, Gallivan S, Sherlaw-Johnson C, Drummond M (2007) Estimating the longterm impact of a prophylactic human papillomavirus 16/18 vaccine on the burden of cervical cancer in the UK. Br J Cancer 96:143-150

Kulasingam SL, Myers ER (2003) Potential health and economic impact of adding a human papillomavirus vaccine to screening programs. J Am Med Assoc 290:781-789

Kulasingam SL, Benard S, Barnabas RV, Largeron N, Myers ER (2008) Adding a quadrivalent human papillomavirus vaccine to the UK cervical cancer screening programme: a cost-effectiveness analysis. Cost Effectiveness and Resource Allocation 6:4

Muñoz N, Bosch F, Castellasague X, Díaz M, de Sanjose $\mathrm{S}$, Hammouda D, Shah K, Meijer C (2004a) Against which human paillomavirus types shall we vaccinate and screen? Int $\mathrm{J}$ Cancer $111: 278-285$

Muñoz N, Mendez F, Posso H, Molano M, van den Brule AJ, Ronderos M, Meijer C, Munoz A (2004b) Incidence, duration, and determination of cervical human papillomavirus infection in a cohort of Columbian women with normal cytological results. J Infect Dis 190:2077-2087
National Board of Health, Danish Centre for Health Technology Assessement (2007) Reduction in the risk of cervical cancer by vaccination against human papillomavirus (HPV) - a health technology assessment. In: National Board of Health, Danish Centre for Health Technology Assessment, Copenhagen

Neilson A, Freiesleben de Blasio B (2007) Economic evaluation of the human papillomavirus (HPV)-vaccination in Norway. In: Norwegian Knowledge Centre for the Health Services, Oslo

Nielsen E, Alfsen GC, Feiring B, Skjeldestad FE, Steen R, Saeterdal I (2007) Prophylactic vaccines against human papillomavirus. In: Norwegian Knowledge Centre for the Health Services, Oslo

Piso B, Wild C (2008) Rational vaccination policy - decision support. Review of international literature for 'rational' vaccination policies. In: Ludwig Boltzmann Institute for Health Technology Assessment, Wien

Roberts C, Tadesse A, Sands J, Halvorsen T, Schonfield T, Dalen A, Skjeldestad F, Jansen K (2006) Detection of HPV in Norwegian cervical biopsy specimens with type-specific PCR and reverse line blot assays. Clinical Virology 36:277-282

Sanders G, Taira AV (2003) Cost effectiveness of a potential vaccine for human papillomavirus. Emerg Infect Dis 9:37-48

Statistik Austria (2007a) Bevölkerungsstatistik. Einzelauswertung. In: Statistik Austria, Wien

Statistik Austria (2007b) Jahrbuch der Gesundheitsstatistik 2005. In: Statistik Austria, Wien

Taira AV, Neukermans CP, Sanders GD (2004) Evaluating human papillomavirus vaccination programs. Emerg Infect Dis 10:1915-1923

Thiry N, Lambert M-L, Cleemput I, Huybrechts M, Neyt M, Hulstaert F, De Laet C (2007) HPV vaccination for the prevention of cervical cancer in Belgium: health technology assessment. In: Belgian Health Care Knowledge Centre (KCE), Brussels

Vutuc C, Haidinger G, Waldhoer T, Ahmad F, Breitenecker G (1999) Prevalence of self-reported cervical cancer screening and impact on cervical cancer mortality in Austria. Wien Klin Wochenschr 111:354-359

Zechmeister I, Freiesleben de Blasio B, Garnett G, Neilson A, Siebert U (2009) Cost-effectiveness analysis of human papillomavirusvaccination programs to prevent cervical cancer in Austria. Vaccine 27:5133-5141 